\title{
EFEITO DA GRAMATURA SOBRE A RESISTÊNCIA AO CISALHAMENTO DA LINHA DE COLA DE DUAS MADEIRAS TROPICAIS: SERU (Allantoma lineata) E MARUPÁ (Simarouba amara)
}

\author{
Cristiane Moreira Tavares dos Santos ${ }^{1}$, Cláudio Henrique Soares Del Menezzi ${ }^{2}$ \\ ${ }^{1}$ Enga ${ }^{a}$ Florestal, Mestranda em Ciências Florestais, UNB, Brasília, DF, Brasil - crismtsantos@yahoo.com.br \\ ${ }^{2}$ Eng. Florestal, Dr., Depto. de Eng. Florestal, UNB, Brasília, DF, Brasil - cmenezzi@unb.br \\ Recebido para publicação: 16/01/2009 - Aceito para publicação: 26/06/2009
}

\begin{abstract}
Resumo
Este trabalho teve como objetivo principal estudar a influência da gramatura do adesivo resorcinolformaldeído na união de peças de madeira, visando à produção de madeira laminada colada (MLC) de duas espécies, seru (Allantoma lineata) e marupá (Simarouba amara), e da mistura entre elas. Foram realizados testes de cisalhamento na linha de cola conforme a NBR 7190 (1997), bem como foi calculada a percentagem de falha na madeira para as duas espécies e para dois tipos de mistura entre elas nas gramaturas 200, 300 e 400 g.m², além de testes de cisalhamento longitudinal das madeiras sólidas. As espécies e misturas se correlacionam com a gramatura através de um modelo quadrático, no qual a maior resistência foi obtida próximo à gramatura de 300 g. $\mathrm{m}^{-2}$, não sendo significativo ao nível de $5 \%$ de probabilidade apenas para a mistura na qual a madeira de seru recebeu o esforço direto da prensa. Os resultados mostraram que a ligação adesivo-madeira foi mais eficiente para a espécie marupá, de baixa densidade. Entretanto, a maior média de resistência ao cisalhamento $(9,46 \mathrm{MPa})$ foi obtida para a colagem da espécie seru. Para ambas as espécies, as gramaturas 300 e $400 \mathrm{~g} . \mathrm{m}^{-2}$ atingiram o requisito da NBR 7190, que estabelece que a eficiência do adesivo deve ser superior a 90\%. Os resultados indicaram a possibilidade de mistura de ambas as espécies.

Palavras-chave: Madeira laminada colada; gramatura; Allantoma lineata; Simarouba amara.
\end{abstract}

\begin{abstract}
Effect of the spread rate on glue shear strength of two tropical hardwoods: seru (Allantoma lineata) and marupá (Simarouba amara). This study aimed at studying the effect of the glue spread rate on bonding strength of two tropical woods: Seru (Allantoma lineata) and Marupá (Simarouba amara). All the shear tests were acomplished according to the Brazilian Design Code NBR 7190 (ABNT, 1997), for each specie and on two kinds of mixtures of these species. For both species they were also done tests to verify the parallel shear strength in solid woods. Using these tests, they were determined the shear strength and the percentage of wood failure for each specie and for their mixtures, using the followings amounts of glue: 200,300 and $400 \mathrm{~g} . \mathrm{m}^{-2}$. Afterwards, the best statistic obtained model, relating the amounts of glue to the woods cited above, was the quadratic model. The best shear strength was obtained around $300 \mathrm{~g} \cdot \mathrm{m}^{-2}$ and was considered not significant at the level of $5 \%$ of probability only for a unique kind of mixture, where Seru receives direct tension of the press machine. The highest shear strength average, 9,46 MPa, was obtained for the Seru wood bonding junction. For both species the amounts of glue 300 and 400 g.m $\mathrm{m}^{-2}$ met the NBR 7190 requirement, which states that the efficiency of the adhesive strength must be above $90 \%$ in comparison to the solid wood. Keywords: Glued-laminated timber; glue amount; Allantoma lineate; Simarouba amara.
\end{abstract}

\section{INTRODUÇÃO}

É comum em todo o mundo a utilização de estruturas de madeira na construção civil, sendo considerado o material de construção mais antigo, devido à sua disponibilidade e facilidade de manuseio. Em geral, essas estruturas são usadas em telhados, vigas, revestimentos, pontes e embarcações, entre outras funções. A madeira é muito utilizada por suas características estéticas, de durabilidade, de facilidade de ser trabalhada e de baixa densidade quando comparada a outros materiais, porém uma de 
suas limitações para uso estrutural é a dificuldade em realizar ligações de uma peça a outra, ou a outros materiais.

Pocius (2002) afirma que uma das vantagens do uso de adesivos em relação a métodos mecânicos tradicionais, como pregos, rebites e parafuso, é que o adesivo dispensa a necessidade de se fazer furos no material a ser unido, evitando assim a concentração de tensões, que pode causar uma diminuição de suas propriedades físicas e mecânicas. Devido à sua porosidade natural, a aplicação adequada do adesivo permitirá sua penetração na superfície da madeira, produzindo assim uma ligação adesiva forte (FAHERTY; WILLIAMSON, 1997).

A madeira laminada colada (MLC) consiste na união de duas ou mais camadas de madeira pelo uso de um adesivo. As lâminas de madeira são selecionadas previamente e preparadas com suas fibras em direção paralela, no sentido de seu comprimento. A espessura máxima permitida das lâminas é de até 5 $\mathrm{cm}$, podendo ser unidas por topo-base, borda-borda e face-face (USDA, 1999). Segundo Pfeil; Pfeil (2003), a MLC apresenta diversas vantagens quando comparada à madeira maciça, tais como a confecção de peças de maior dimensão, a redução dos defeitos ocasionados por secagem irregular, a possibilidade de fazer construções de peças de eixo curvo e por ser possível selecionar a qualidade das lâminas para as posições de maior tensão. Por outro lado, a MLC apresenta como desvantagens um alto custo quando comparado à madeira maciça e limitações de transporte.

As estruturas geradas através das técnicas de colagem de madeira laminada dependem de diversos parâmetros, como o tipo e a quantidade apropriada de adesivo, a escolha apropriada da espécie de madeira (levando em conta densidade e propriedades mecânicas), a direção paralela das fibras das lâminas para a montagem da MLC e da umidade da madeira no momento de colagem. Para a fabricação de MLC, devem ser usados apenas adesivos estruturais resistentes às intempéries. Reis; Jesus (2008) afirmam que, entre os tipos de adesivos, o mais aplicado atualmente em estruturas é o adesivo resorcinolformaldeído, termofixo. Segundo Broughton; Hutchinson (2000), os adesivos termofixos são muito usados por apresentarem boa adesão a outros materiais e por serem tolerantes a variações de umidade. Faherty; Williamson (1997) descrevem os adesivos sintéticos termofixos como aqueles que, uma vez polimerizados, tornam-se insolúveis e não fundíveis, quando um catalisador adequado é utilizado.

A Norma Técnica Brasileira de Estruturas de Madeira-NBR 7190 (ABNT, 1997) permite ligações de madeira através do uso de adesivos apenas para a fabricação de juntas longitudinais de MLC, nas quais as madeiras devem ser previamente secas, ao ar ou em estufa. A mesma norma também estabelece três ensaios para a caracterização da madeira para essa finalidade: o cisalhamento na linha de cola, a tração normal à linha de cola e a resistência de emendas dentadas e biseladas. A resistência da junta colada deve ser no mínimo igual ao cisalhamento longitudinal da madeira. As ligações produzidas pela cola devem ter resistência e durabilidade garantidas para toda a vida útil da estrutura (ABNT, 1997). A escolha apropriada da gramatura, quantidade de adesivo por $\mathrm{m}^{2}$ de madeira a ser colada, deve levar em conta que os adesivos possuem custo elevado e interferem na resistência e qualidade das ligações. Segundo Faherty; Williamson (1997), a utilização de testes de cisalhamento na linha de colagem é a maneira mais eficaz de testar a resistência das ligações realizadas por adesivos.

Lobão; Gomes (2006) fizeram ensaios com madeiras de eucalipto com a gramatura de $300 \mathrm{~g} . \mathrm{m}^{-2}$ de resorcinol-formaldeído em face dupla e pressão de $1 \mathrm{MPa}$. Para os corpos de prova colados com madeiras de alta densidade e com as fibras paralelas entre si, a resistência média encontrada foi de 9,7 MPa (madeira a 12\% de umidade). Vital et al. (2006) fizeram ensaios com juntas de três regiões do tronco de Eucalyptus saligna, Eucalyptus grandis e Pinus elliottii, também utilizando gramatura de 300 g. $\mathrm{m}^{-2} \mathrm{em}$ face dupla para a colagem com o adesivo resorcinol-formaldeído, e $150 \mathrm{~g} . \mathrm{m}^{-2}$ em face simples para o adesivo poliacetato de vinila. Para o resorcinol-formaldeído a média encontrada da tensão de ruptura foi de 7,7 MPa, e para o adesivo poliacetato de vinila (PVA) de média e alta viscosidade a média foi de 8,2 MPa e 7,9 MPa, respectivamente (corpos de prova a 14\% de umidade), sendo o Eucalyptus saligna a espécie que apresentou os valores médios mais elevados de resistência ao cisalhamento e os menores valores de falha na madeira.

Para a fabricação da MLC, é ideal que sejam utilizadas madeiras de densidade entre 0,35 e $0,7 \mathrm{~g} / \mathrm{cm}^{3}$. Seru (Allantoma lineata) e marupá (Simarouba amara) são duas espécies bastante comercializadas no Distrito Federal e possuem densidade de 0,60 e $0,37 \mathrm{~g} / \mathrm{cm}^{3}$, respectivamente. Para a fabricação de MLC, é aceitável a colagem de tábuas de mesma espécie ou de espécies diferentes em sua estrutura, podendo-se empregar lâminas de maior resistência nas regiões de maior tensão. Lobão; Gomes (2006) relataram em seu trabalho, realizado com madeiras de baixa densidade, de alta densidade ou misturando duas espécies de densidades diferentes, que a colagem de madeiras de densidades muito diferentes produziu juntas com resistência menor que a da madeira menos densa, ocorrendo ruptura 
sempre na madeira de menor densidade. Os testes foram feitos com as fibras em direção paralela e perpendicular entre si, sempre havendo um grande percentual de falhas encontrado na madeira, comprovando uma boa qualidade da adesão.

River et al. (1991) afirmam que, mesmo utilizando-se um único tipo de madeira para fabricação de vigas, propriedades distintas podem ser obtidas. A qualidade, a espessura e o número de tábuas podem variar para se obter a resistência desejada, assim como a escolha da espécie adequada de madeira e a quantidade de adesivo utilizado. De acordo com Petrauski et al. (2006), quando consideradas as circunstâncias de mercado, o uso de mais de uma espécie em peças de madeira laminada colada pode contribuir para diminuição de custos de fabricação, além de aumentar a possibilidade de peças diferentes esteticamente. Para tanto, no uso estrutural, deve-se pressupor uma colagem resistente entre as espécies envolvidas. A qualidade das ligações adesivas em MLC está relacionada, entre outros fatores, à densidade da madeira. Vital et al. (2006) afirmam que madeiras menos densas são mais porosas e permitem uma maior permeabilidade do adesivo, porém em geral são menos resistentes a esforços. Devido à melhor colagem do adesivo em madeiras menos densas, costuma-se utilizar espécies de densidade inferior a $0,7 \mathrm{~g} / \mathrm{cm}^{3}$ e superior a $0,35 \mathrm{~g} / \mathrm{cm}^{3}$, para não comprometer a resistência mecânica das estruturas. Afirmam também que as folhosas geralmente apresentam maior dificuldade de colagem que as coníferas, isso devido à sua constituição química e sua maior complexidade estrutural. Ademais, características intrínsecas da madeira podem interferir na densidade e porosidade, na variação de cerne para alburno e entre lenhos inicial e tardio, devido à presença de extrativos nos elementos anatômicos, interferindo significativamente dessa forma no processo de colagem (MARRA, 1992).

O presente estudo teve como objetivo estudar o efeito da gramatura sobre a resistência de ligações coladas de duas espécies de madeiras tropicais e inferir, a partir dos dados, a possibilidade de utilização das espécies Simarouba amara e Allantoma lineata para a fabricação de MLC, utilizando-se uma única espécie ou a mistura de ambas.

\section{MATERIAL E MÉTODOS}

Inicialmente, foram adquiridas em mercado local pranchas aplainadas de madeira de $300 \times 12 \times$ $5,3 \mathrm{~cm}^{3}$ de seru (Allantoma lineata) e marupá (Simarouba amara), cuja identificação foi feita macroscopicamente por meio de comparação com o material depositado na Xiloteca (Index Xilarium FPBw) do Laboratório de Produtos Florestais (LPF). As espécies escolhidas para este experimento são amplamente comercializadas nas madeireiras do Distrito Federal e possuem densidade inferior a $0,7 \mathrm{~g} / \mathrm{cm}^{3}$ a $12 \%$ de umidade, valor considerado limite de densidade das madeiras usualmente utilizadas na produção de MLC. Em seguida, as pranchas foram dispostas em câmara climática $\left(65 \% ; 20{ }^{\circ} \mathrm{C}\right)$ até massa constante. Após a climatização, as pranchas foram desdobradas nas dimensões 150 x $5 \times 5 \mathrm{~cm}^{3} \mathrm{e}$ coladas com o adesivo à base de resorcinol-formaldeído (Cascophen RS, Alba Química S.A), de modo que houvesse a combinação de pranchas de mesma espécie e de espécies distintas, nas gramaturas de 200, 300 e 400 g.m ${ }^{-2}$. O adesivo foi aplicado de forma manual e em face simples. Feita a colagem, as madeiras foram reenviadas para a sala de climatização por um período de duas semanas. A preparação do adesivo foi realizada segundo as instruções do fabricante, ou seja, uma parte em massa do catalisador para cinco partes em massa da resina. O delineamento experimental é apresentado na tabela 1. Foram avaliados ao todo 12 tratamentos, sendo seis com única espécie (T1 a T6) e seis com mistura entre as espécies (T7 a T12). Para cada tratamento foram feitos 12 corpos de prova (repetições), totalizando assim 144 CPs. A confecção dos CPs seguiu as prescrições da norma NBR 7190 (1997). A figura 1 ilustra os CPs confeccionados e a forma de ensaio para cada tratamento.

A determinação da resistência ao cisalhamento da linha de cola $\left(\mathrm{f}_{\mathrm{gv}, 0}\right)$ foi conduzida segundo as recomendações da norma ASTM D905 (Figura 2), uma vez que o equipamento não possuía regulagem para a velocidade de ensaio de $2,5 \mathrm{MPa} / \mathrm{min}$, conforme prescreve a NBR 7190. Doze corpos de prova de madeira sólida para cada espécie foram utilizados para a determinação da resistência ao cisalhamento paralelo $\left(\mathrm{f}_{\mathrm{v}, 0}\right)$, de modo a avaliar a eficiência do adesivo (E\%), segundo Equação 1.

$$
E \%=\frac{f_{g v, 0}}{f_{v, 0}} \times 100>90 \%
$$

Na qual: $\mathrm{f}_{\mathrm{gv}, 0}=$ resistência ao cisalhamento da linha de cola, MPa;

$\mathrm{f}_{\mathrm{v}, 0}=$ resistência ao cisalhamento longitudinal da madeira sólida, $\mathrm{MPa}$. 
Tabela 1. Delineamento experimental.

Table 1. Experimental design.

\begin{tabular}{lccc}
\hline Tratamento & $\begin{array}{c}\text { Peça que recebe carga no } \\
\text { ensaio }(\mathbf{A})\end{array}$ & $\begin{array}{c}\text { Peça que reage à carga } \\
\text { aplicada }(\mathbf{B})\end{array}$ & Gramatura $\left(\mathbf{g} \cdot \mathbf{m}^{-\mathbf{2}}\right)$ \\
\hline T1 & Seru & Seru & 200 \\
T2 & & & 300 \\
T3 & Marupá & Marupá & 400 \\
\hline T4 & & & 200 \\
T5 & & & 300 \\
T6 & Seru & 400 \\
\hline T7 & & & 200 \\
T8 & & & 300 \\
T9 & & Marupá & 400 \\
\hline T10 & & & 200 \\
T11 & Seru & 300 \\
T12 & & & 400 \\
\hline
\end{tabular}
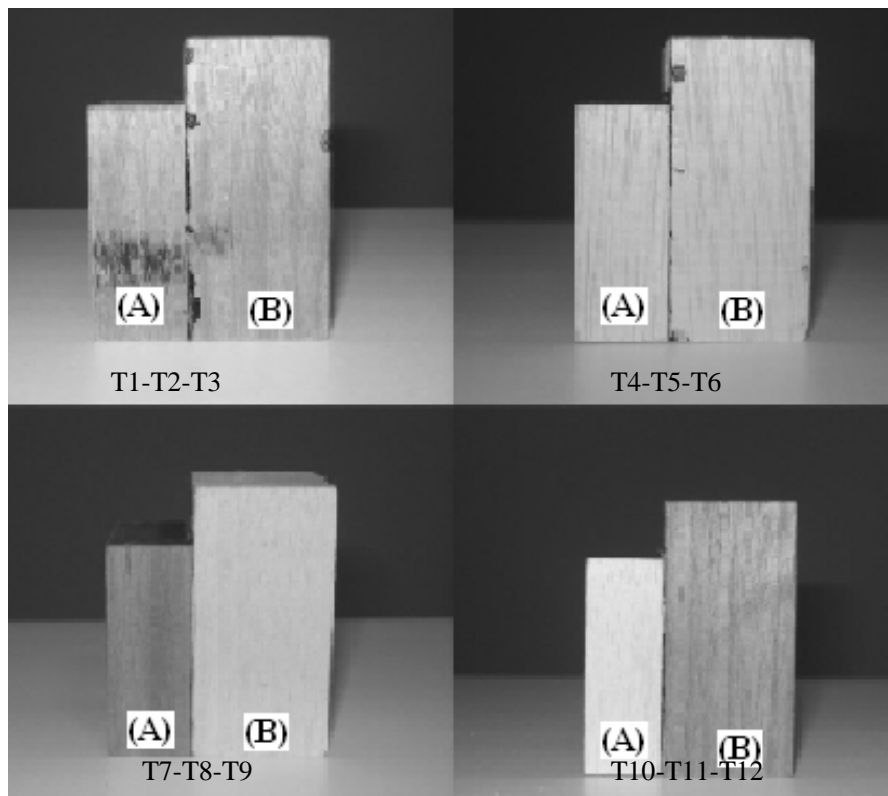

Figura 1. Corpos de prova dos tratamentos avaliados. (A: peça que recebe carga no ensaio; B: peça que reage à carga de ensaio).

Figure 1. Samples from the evaluated treatments. (A: side which load was applied; B: side which load was reacted).
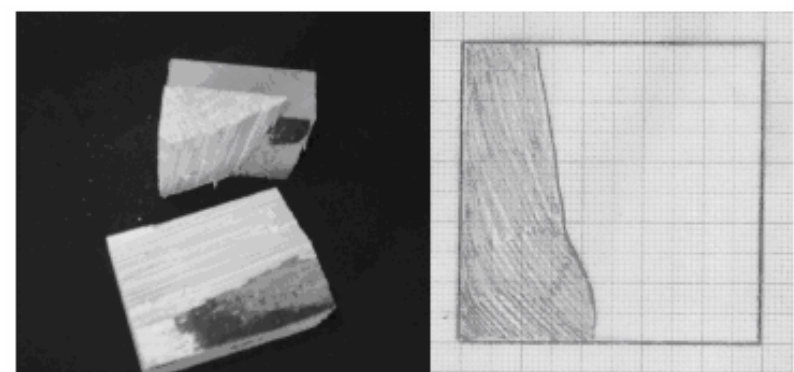

Falha na linha de cola

Falha na madeira

Figura 2. Procedimento para avaliação da porcentagem de falha na madeira.

Figure 2. Procedure to evaluate the wood failure. 
Após a realização dos ensaios de fgv, 0 e fv, 0 , os CPs foram secos em estufa para a determinação do teor de umidade. Os valores de $\mathrm{f}_{\mathrm{gv}, 0}$ e $\mathrm{f}_{\mathrm{v}, 0}$ foram corrigidos para a umidade padrão de $12 \%$, segundo ABNT (1997). Para cada tratamento e para as duas espécies, foi determinado, conforme indica a norma supracitada, o valor característico $X_{k}$. A análise da porcentagem de área cisalhada na linha de cola foi realizada com o auxílio de um papel milimetrado $(5 \mathrm{~cm}$ x $5 \mathrm{~cm})$, sendo possível, a partir da contagem dos espaços, analisar a porcentagem de área cisalhada na linha de cola e a cisalhada na própria madeira (Figura 2)

A análise estatística foi realizada com o uso do programa SPSS 13.0 e foi feita em duas etapas. $\mathrm{Na} 1^{\mathrm{a}}$ etapa, procurou-se avaliar todos os tratamentos em conjunto, bem como o efeito isolado das espécies e da mistura sobre a resistência ao cisalhamento paralelo da linha de cola. $\mathrm{Na} 2^{\mathrm{a}}$ etapa, procurouse avaliar diretamente o efeito da gramatura para cada espécie e mistura testada. Em ambas as etapas, foram empregadas a análise de regressão e o teste de Tukey a 5\% de probabilidade.

\section{RESULTADOS E DISCUSSÃO}

As médias de resistência ao cisalhamento da linha de cola para cada tratamento encontram-se na tabela 2. Os valores apresentados já estão corrigidos para o teor de umidade de $12 \%$. A análise estatística feita através do teste de Tukey indicou quatro grupos diferentes significativamente entre os tratamentos, sendo encontrados maiores valores de resistência para os tratamentos T2 e T3.

Tabela 2. Valores médios da resistência ao cisalhamento paralelo da linha de cola para os tratamentos avaliados.

Table 2. Mean values of the glue shear strength for the different evaluated treatments.

\begin{tabular}{lc}
\hline Tratamento & $\mathbf{f}_{\mathbf{g v}, \mathbf{0}}(\mathbf{M P a})$ \\
\hline T2 & $10,64 \mathrm{a}$ \\
T3 & $10,26 \mathrm{a}$ \\
T5 & $8,23 \mathrm{~b}$ \\
T8 & $8,19 \mathrm{~b}$ \\
T6 & $7,61 \mathrm{~b}$ \\
T1 & $7,48 \mathrm{bc}$ \\
T11 & $7,18 \mathrm{bc}$ \\
T9 & $7,12 \mathrm{bc}$ \\
T12 & $6,93 \mathrm{bc}$ \\
T10 & $6,06 \mathrm{~cd}$ \\
T4 & $5,53 \mathrm{~d}$ \\
T7 & $5,26 \mathrm{~d}$ \\
\hline Nota: Em cada coluna, médias seguidas pelas mesmas letras não se diferenciam \\
estatisticamente pelo teste de Tukey ao nível de 1\% de probabilidade. T1: seru-seru, 200 g.m-2 \\
T2: seru-seru, 300; T3: seru-seru, 400; T4: marupá- marupá, 200; T5: marupá-marupá, 300; \\
T6: marupá-marupá, 400; T7: marupá-seru, 200; T8: marupá-seru, 300; T9: marupá-seru, 400; \\
T10: seru-marupá, 200; T11: seru-marupá, 300; T12: seru-marupá, 400.
\end{tabular}

Quando avaliado apenas o efeito da espécie e da mistura, foram encontrados dois grupos diferentes significativamente. A colagem de seru-seru apresentou maior resistência ao cisalhamento na linha de cola (Tabela 3). Para a colagem de marupá-marupá ou as misturas de marupá-seru não houve diferenças significativas, indicando que a mistura pode ser feita sem perda de resistência.

Tabela 3. Efeito da espécie e da mistura sobre a resistência ao cisalhamento paralelo de linha de cola. Table 3. Effect of wood and wood species mixture on the glue shear strength.

\begin{tabular}{lcc}
\hline Peças A e B & $\mathbf{N}^{\mathbf{0}} \mathbf{C P s}$ & $\left.\mathbf{f}_{\mathbf{g v}, \mathbf{0}} \mathbf{( M P a}\right)$ \\
\hline 1 (seru-seru) & 36 & $9,46 \mathrm{a}$ \\
2 (marupá-marupá) & 36 & $7,12 \mathrm{~b}$ \\
3 (marupá-seru) & 35 & $6,85 \mathrm{~b}$ \\
4 (seru-marupá) & 36 & $6,72 \mathrm{~b}$ \\
\hline Nota: Médias seguidas pelas mesmas letras não se diferenciam estatisticamente, pelo teste de Tukey a 5\% de probabilidade.
\end{tabular}


Na figura 3, pode-se observar que existe interação entre espécie e gramatura, já que a curva de seru-marupá intercepta a curva de marupá-marupá e marupá-seru, comprovando que espécies ou mistura entre espécies diferentes respondem de maneira diferente ao aumento ou redução da quantidade de adesivo utilizado. A colagem de marupá-marupá, seru-seru e marupá-seru são muito mais afetadas por variações na gramatura que a de seru-marupá.

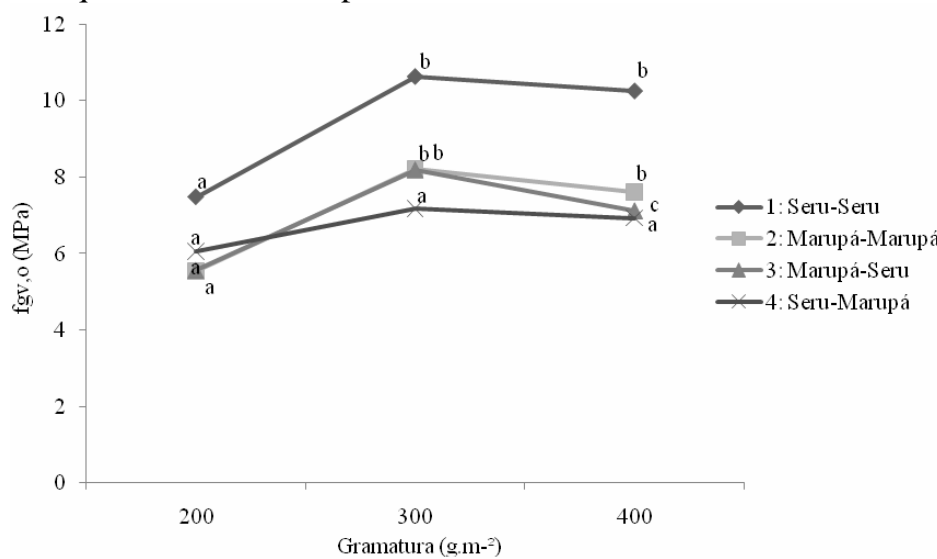

Nota: Para cada curva, pontos seguidos pelas mesmas letras não se diferenciam estatisticamente, pelo teste de Tukey a $5 \%$ de probabilidade.

Figura 3. Efeito combinado entre espécie e gramatura. (1: seru-seru; 2: marupá-marupá; 3: marupáseru; 4: seru-marupá).

Figure 3. Combined effect of wood species and glue rate spread.

A avaliação do efeito da gramatura, independentemente da espécie, indica a tendência de um modelo quadrático (Figura 4). Observou-se no presente trabalho que as três gramaturas avaliadas apresentam médias diferentes significativamente. $\mathrm{O}$ baixo valor de $\mathrm{R}^{2}$ pode ser explicado pela grande dispersão dos dados, visto que a figura 4 apresenta todos os $144 \mathrm{CPs}$ avaliados. Apesar do $\mathrm{R}^{2}$ ser considerado baixo, a regressão foi significativa a $5 \%$ de probabilidade e foi mantida para mostrar a tendência observada para os dados.

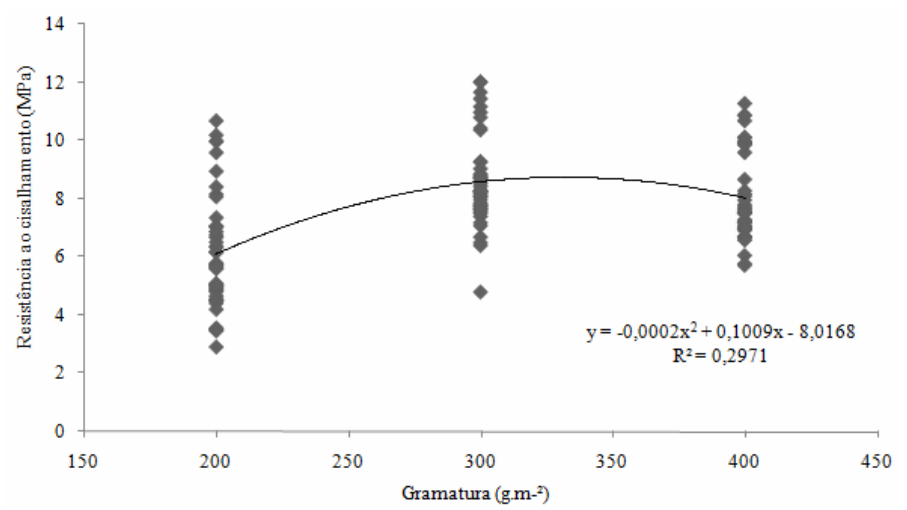

Figura 4. Efeito da gramatura sobre a resistência ao cisalhamento da linha de cola $(\mathrm{N}=144)$.

Figure 4. Effect of glue rate spread on the shear glue strength for all evaluated treatments $(\mathrm{N}=144)$.

A figura 5 apresenta os resultados para as espécies e as misturas estudadas. A análise de regressão indica a tendência de um modelo quadrático para a colagem de marupá-marupá, seru-seru e para a mistura marupá-seru. Para esses tratamentos, de um modo geral, a resistência ao cisalhamento teve um valor máximo na gramatura de $300 \mathrm{~g} . \mathrm{m}^{-2}$, e não houve qualquer aumento da resistência quando se aumentou a gramatura para $400 \mathrm{~g} . \mathrm{cm}^{-2}$. No entanto, para a mistura marupá-seru, todas as gramaturas estudadas foram diferentes significativamente entre si. O maior valor de resistência ao cisalhamento se deu para a gramatura de 300 g.m $\mathrm{m}^{-2}$, e a menor resistência foi observada em 200 g.m ${ }^{-2}$. Para a colagem de 
seru-marupá, o teste de Tukey indicou não haver diferença significativa entre gramaturas variando de 200 a 400 g.m². A baixa correlação entre os dados obtidos pela análise de regressão confirma esse resultado. Nesse caso, recomenda-se a utilização de $200 \mathrm{~g}$ de cola por $\mathrm{m}^{2}$ de madeira, visando à economia do material adesivo e a consequente redução de gastos para a produção de MLC.
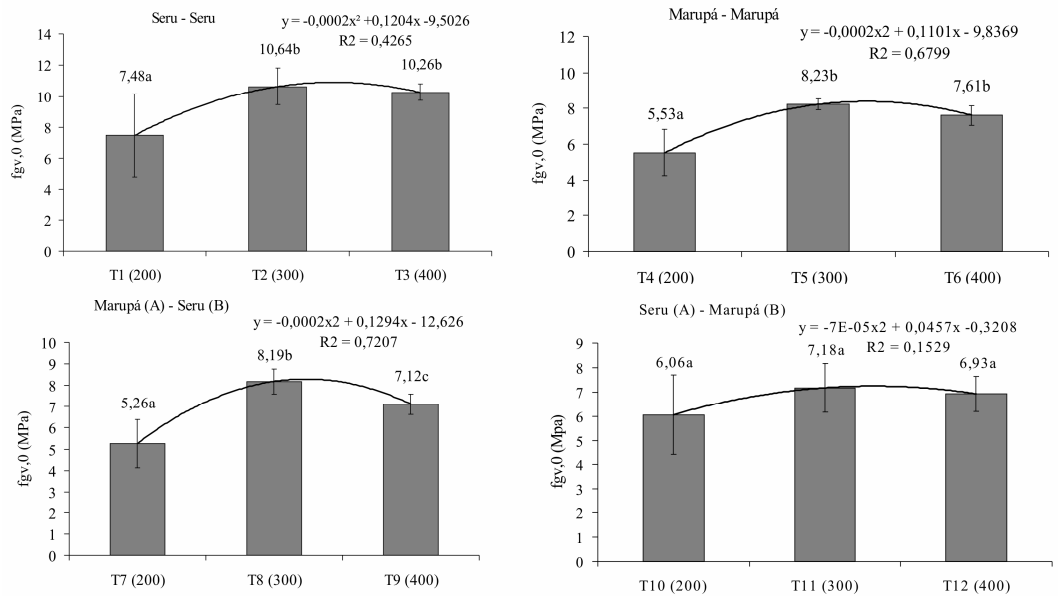

Nota: Em um mesmo gráfico, médias seguidas pelas mesmas letras não diferem entre si pelo teste de Tukey, a 5\% de probabilidade. Figura 5. Efeito da gramatura sobre a resistência da linha de cola para as espécies e as misturas.

Figure 5. Effect of the glue rate spread on the wood and wood species mixtures values of shear glue strength.

A recomendação usual de utilização do adesivo é de 350 g.m², porém esse valor, em todas as espécies e misturas estudadas, poderia ser reduzido para $300 \mathrm{~g} . \mathrm{m}^{-2} \mathrm{sem}$ afetar negativamente a resistência, visto que esse valor não apresenta diferença estatística significativa em relação ao valor recomendado, otimizando economicamente a produção. A norma brasileira de projeto de estruturas de madeira, NBR 7190 (1997), leva em consideração o valor de resistência característico que é empregado na determinação dos valores de cálculo de dimensionamento e/ou verificação dessas estruturas. Os valores apresentados na figura 6 encontram-se corrigidos para o teor de $12 \%$ de umidade.

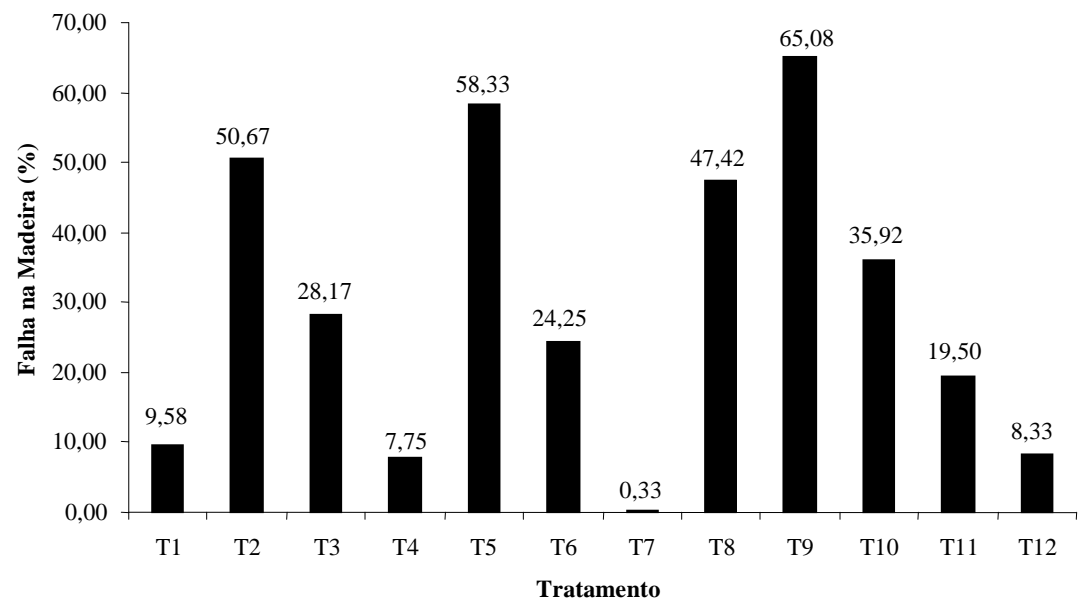

Figura 6. Valor característico para os 12 tratamentos e da madeira sólida. T1: seru-seru, 200 g.m ${ }^{-2}$; T2: seru-seru, 300; T3: seru-seru, 400; T4: marupá-marupá, 200; T5: marupá-marupá, 300; T6: marupá-marupá, 400; T7: marupá-seru, 200; T8: marupá-seru, 300; T9: marupá-seru, 400; T10: seru-marupá, 200; T11: seru-marupá, 300; T12: seru-marupá, 400.

Figure 6. Characteristic values of the evaluated treatments and solid wood. 
Apesar do seru apresentar maior resistência ao cisalhamento na linha de cola, o adesivo à base de resorcinol-formaldeído mostrou-se mais eficiente para a madeira marupá, de menor densidade. Para ambas as espécies, apenas a gramatura de 200 g.m² não satisfez a norma NBR 7190, que estabelece que a eficiência do adesivo deve ser maior que $90 \%$ (Figura 7).

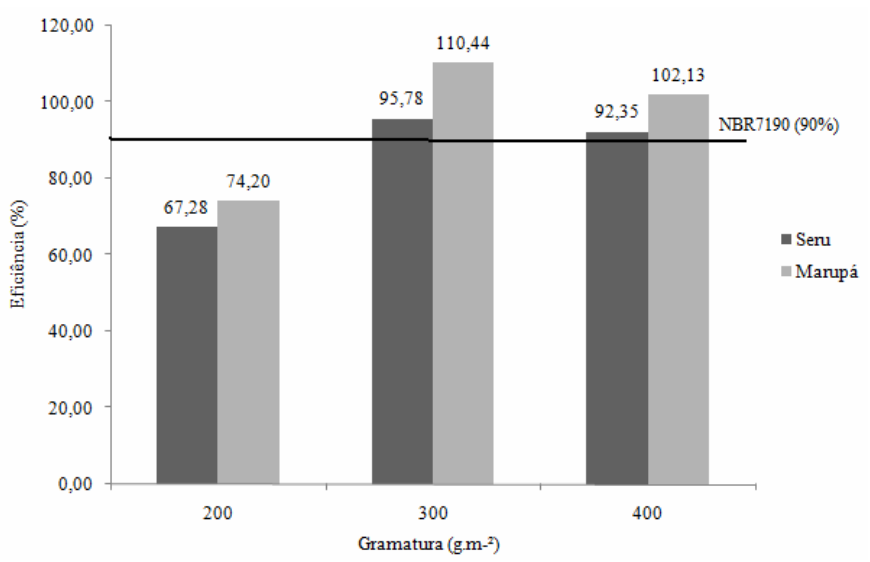

Figura 7. Eficiência do adesivo de acordo com a gramatura utilizada.

Figure 7. Adhesive efficiency according to the glue rate spread.

A eficiência do adesivo para as gramaturas de 300 e 400 g.m $\mathrm{m}^{-2}$ nas duas espécies estudadas foi superior aos valores obtidos por Macedo et al. (2008a) em outras três espécies de madeira da Amazônia, também de densidade baixa e leve. As médias de eficiência da lâmina de cola para o mesmo adesivo na gramatura variando de 350 a 400 g.m ${ }^{-2}$ foi de $87 \%, 83 \%$ e $74 \%$ para as espécies Couratani guianenses (tauari), Couratani oblongifolia (tauari 2) e Qualea cf. lancifolia (mandioqueira), respectivamente.

O percentual de falha na madeira para os diversos tratamentos encontra-se na figura 8. Pode-se inferir que um maior percentual de falha na madeira está relacionado com uma maior eficiência do adesivo. Os tratamentos que obtiveram melhores resultados de falha na madeira são: T2, para a colagem de seru-seru; T5, para marupá-marupá; T9, para marupá-seru; e T10, para seru-marupá. O percentual de falha na madeira pode apresentar grandes variações para uma mesma espécie. Lobão; Gomes (2006) obtiveram a porcentagem média dessas falhas variando de 69 a $84 \%$ para madeiras de Eucaliptus sp. de diferentes densidades e em dois diferentes planos (fibras perpendiculares e paralelas). $\mathrm{O}$ adesivo utilizado foi também o resorcinol-formaldeído, na gramatura de 300 g.m $\mathrm{m}^{-2}$, em face dupla. Porém Lima et al. (2008) obtiveram o percentual de falha na madeira variando de 29 a $64 \%$ para quatro clones de Eucalyptus, para o mesmo adesivo, na gramatura de 250 g.m $\mathrm{m}^{-2}$, em linha dupla.

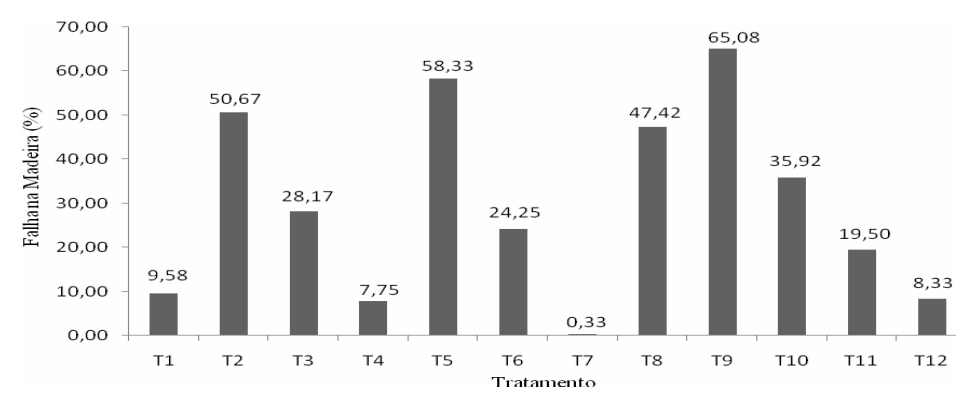

Figura 8. Percentual de falha na madeira. T1: seru-seru, 200 g.m²; T2: seru-seru, 300; T3: seru-seru, 400; T4: marupá-marupá, 200; T5: marupá-marupá, 300; T6: marupá-marupá, 400; T7: marupá-seru, 200; T8: marupá-seru, 300; T9: marupá-seru, 400; T10: seru-marupá, 200; T11: seru-marupá, 300; T12: seru-marupá, 400.

Figure 8. Wood failure percentual. 
Os resultados de porcentagem de falha na madeira obtidos neste trabalho foram inferiores aos encontrados por Macedo et al. (2008b) para o cisalhamento na lâmina de cola de marupá. Os autores utilizaram o adesivo Cascophen RS na gramatura variando de 350 a 400 g. $\mathrm{m}^{-2}$ e obtiveram valores de porcentagem de falha na madeira para diferentes pressões de colagem de $46 \%\left(6 \mathrm{~kg} \cdot \mathrm{cm}^{-2}\right), 94 \%\left(8 \mathrm{~kg} \cdot \mathrm{cm}^{-2}\right)$ e $85 \%\left(10 \mathrm{~kg} . \mathrm{cm}^{-2}\right)$. O percentual médio de falha na madeira de todos os tratamentos foi de $29,61 \%$, e para cada gramatura a média foi de $13,40 \%\left(200\right.$ g. $\left.\mathrm{m}^{-2}\right), 43,98 \%\left(300 \mathrm{~g} . \mathrm{m}^{-2}\right)$ e $31,45 \%$ (400 g.m $\left.\mathrm{m}^{-2}\right)$. O baixo percentual médio para cada gramatura indica um problema na adesão entre a madeira e o adesivo. Apesar das gramaturas de 300 e 400 g.m ${ }^{-2}$ satisfazerem as condições da NBR 7190 (1997), esses valores de eficiência poderiam ser melhorados com a observância de alguns fatores, entre os quais um estudo das propriedades químicas das espécies marupá e seru, o controle da pressão na hora da colagem das tábuas de madeira, a utilização de linha de cola dupla, ao invés de simples, como foi realizado, e a realização de tratamentos na madeira, como lixá-la antes da colagem. De acordo com River et al. (1991), dependendo das propriedades químicas na superfície da madeira, faz-se necessário o uso de tratamentos antes da colagem, tais como aplicação de preservativos. A colagem em linha dupla é mais indicada para a produção de MLC que a de linha simples, já que esta é mais afetada pela viscosidade do adesivo e pela rapidezcom que ele se espalha e modifica a superfície da madeira.

\section{CONCLUSÕES}

- A resistência ao cisalhamento da linha de cola foi influenciada pela gramatura e pela espécie utilizada, sendo a gramatura recomendada para ambas as espécies de $300 \mathrm{~g} . \mathrm{m}^{-2}$.

- Os valores de resistência e eficiência de colagem obtidos neste trabalho indicam potencial das espécies Simarouba amara e Allantoma lineata para a fabricação de MLC, podendo inclusive ser utilizada a mistura entre elas.

- O adesivo à base de resorcinol-formaldeído na gramatura de $200 \mathrm{~g} . \mathrm{m}^{-2}$ não se mostrou eficiente para a colagem das espécies estudadas, segundo o que estabelece a NBR 7190 (1997).

- No entanto, dado o baixo percentual de falha na madeira, estudos complementares devem ser realizados no sentido de melhorar a compatibilidade entre as madeiras e o adesivo estudado, o que poderá inclusive aumentar os valores de resistência observados.

\section{AGRADECIMENTOS}

Ao Laboratório de Produtos Florestais (LPF), do Serviço Florestal Brasileiro, onde os ensaios foram realizados, e ao CNPq, pela concessão da bolsa de Iniciação Científica ao primeiro autor.

\section{REFERÊNCIAS}

AMERICAN SOCIETY FOR TESTING AND MATERIALS. Standard Test Method for Strength Properties of Adhesive Bonds in Shear by Compression Loading. ASTM D905-03. 2005.

ASSOCIAÇÃO BRASILEIRA DE NORMAS TÉCNICAS. Projetos de estruturas de madeira. Rio de Janeiro: ABNT 7190. 1997. 107 p.

BROUGHTON, J. G.; HUTCHINSON, A. R. Adhesive systems for structural connections in timber. Int. J. Adhesion and Adhesives. 2001. v. 21, $\mathrm{n}^{\circ}$ 3: 177-186.

FAHERTY, K. F.; WILLIAMSON, G. Wood engineering and construction handbook. 3. ed. New York: McGraw-Hill Publishing Company. 1997.

GUIMARÃES, E. F.; MAUTONE, L.; RIZZINI, C. T.; MATOS FILHO, A. Árvores do Jardim Botânico do Rio de Janeiro. Rio de Janeiro: Jardim Botânico do Rio de Janeiro. 1993. 234 p.

IPT. Informações sobre madeiras. In: Instituto de Pesquisas Tecnológicas. Disponível em <http://www.ipt.br/areas/ctfloresta/lmpd/madeiras/busca/>. Acessado em 16 de janeiro de 2008. 
LIMA, C. K. P.; MORI, F. A.; MENDES, L. M.; TRUGUILHO, P. F.; MORI, C. L. S. Colagem de madeira de clones com três adesivos comerciais. Revista Scientia Florestalis, Piracicaba. 2008. v. 36, n. 77, p. 73-77.

LOBÃO, M. S.; GOMES, A. Qualidade de adesão de eucalipto em corpos de prova colados em dois diferentes planos e densidades. Cerne, Lavras. 2006. v. 12, n. 2, p. 194-200.

LPF. Madeiras Brasileiras. In: Laboratório de Produtos Florestais. Disponível em: <http://www.ibama.gov.br/lpf/madeira>. Acessado em 12 de dezembro de 2007.

MACEDO, A. N.; CALIL JR., C. Cadernos de engenharia de estruturas. São Carlos. 1999. n. 7, p. 1-23.

MACEDO, A. N.; RAMOS, M. A.; FERNANDES, M. C. O.; BENTES, J. L. 2008a. Espécies de madeira da região amazônica: possibilidade de aplicação na tecnologia da madeira laminada colada. XI Encontro Brasileiro em Madeiras e em Estruturas de Madeiras (EBRAMEM).

MACEDO, A. N.; ROSSI, C. R.; MONTEIRO, R. F. L.; SILVA, R. N. 2008b. Efeito da variação da pressão de colagem na resistência ao cisalhamento e tração normal à lâmina de cola para duas espécies de madeira da região amazônica. XI Encontro Brasileiro em Madeiras e em Estruturas de Madeira (EBRAMEM).

MARRA, G. G. Technology of wood bonding: principles in practice. New York. 1992. Van Nostrand Reinhold, 454 p.

OLIVEIRA JÚNIOR, S. F.; AZAMBUJA, M. A.; DIAS, A. A. Análise da viabilidade de aplicação de adesivos alternativos em emenda dentada estrutural para a espécie Eucalyptus grandis. Encontro Brasileiro em Madeiras e em Estruturas de Madeira (EBRAMEM), 10, 1, ISBN: Português, Meio digital. 2006.

PETRAUSKI, S. M. F. C.; GALINA, M.; PETRAUSKI, A. Comportamento mecânico e percentual de falha na madeira de juntas coladas mistas de Eucalyptus e Pinus. X Encontro Brasileiro em Madeiras e em Estruturas de Madeira (EBRAMEM). 2006.

PFEIL, W. E.; PFEIL, M. Estruturas de madeira. 6. ed. Rio de Janeiro. 2003. LTC. 224 p.

POCIUS, A. V. Adhesion and adhesives technology: an introduction. 2. ed. Munich (Alemanha): Hanser. ISBN 3446217312. 2002. 319 p.

REIS, A. L. C.; JESUS, J. M. H. Resistência ao cisalhamento na linha de cola de três espécies de madeira do norte do estado de Mato Grosso para o uso em madeira laminada colada (MLC). XI Encontro Brasileiro em Madeiras e em Estruturas de Madeira (EBRAMEM). 2008.

REMADE. Madeiras brasileiras e exóticas. In: Revista da Madeira. Disponível em: <http://www.remade.com.br/pt/mad_exoticas.php?cat=1>. Acessado em 12 de dezembro de 2007.

RIVER, B. H.; VICK C. B.; GILLESPIE R. H. "Wood as an adherend". In: Treatise on adhesion and adhesives, Vol. 7, Marcel Dekker, Minford, J. D., Ed., New York. 1991. 230 p.

SHIELDS, J. Adhesives handbook. 3. ed. Butter\&Tanner Ltd. UK. 1988. 358 p.

UNITED STATES DEPARTMENT OF AGRICULTURE. Wood handbook: wood as engineering material. USDA: Washington. 1999. 473 p.

VITAL, B. R.; MACIEL, A. S.; LUCIA, R. M. D. Qualidade de Juntas coladas com lâminas de madeira oriundas de três regiões do tronco de Eucalyptus grandis, Eucalyptus saligna e Pinus elliottii. Revista Árvore, Viçosa-MG. 2006. v. 30, n. 4, p. 637-644.

ZANGIÁCONO, A. L. Emprego de espécies tropicais alternativas na produção de elementos estruturais de madeira laminada colada. Tese (Mestrado), Universidade de São Paulo, São Carlos. 2003. 